\title{
Effect of Combined Antibiotics and Biofilm Formation in Some Bacterial Pathogens from Otitis Media among Children in Baghdad, Iraq
}

\author{
Mohammed Khudhair Alwan', Kais Kassim Ghaima², Zainab Shaban Khalaf ${ }^{1}$ \\ ${ }^{1}$ Ministry of Health, Baghdad, Iraq, ${ }^{2}$ Institute of Genetic Engineering and Biotechnology for Postgraduate Studies, \\ University of Baghdad, Baghdad, Iraq
}

\begin{abstract}
Otitis media bacterial infections during childhood may contribute to the development of repeated nasopharyngeal infections and it is complicated of recurrent or chronic middle ear diseases, especially with multidrug resistant strains.

The results of antibiotic synergism for the most prevalent three bacterial species, isolated from otitis media infections in children, and had the highest antibiotic resistance was revealed that combinations ceftazidimeamikacin and ceftazidime- ciprofloxacin displayed synergistic activity fractional inhibitory concentration (FIC $\leq 0.5$ ) against the most of tested isolates; Pseudomonas aeruginosa, Porteus mirabilis, and Klebsiella pneumonia. In the biofilm test all tested isolates were showed biofilm production by tissue culture plate method (TCP), in the present study, the strong biofilm production was most prevalent in Pseudomonas aeruginosa $(100 \%)$ followed by $S$. aureus $(71.4 \%)$. Biofilm formation increases the activity of antibiotics in children otitis media infections and using antibiotics combinations may be essential for optimum management of OM patients.
\end{abstract}

Keywords: Otitis media, Biofilm, Antibiotics, synergistic activity.

\section{Introduction}

Otitis media $(\mathrm{OM})$ is an inflammatory disease of the middle ear, with different medical conditions and symptoms. It is caused as a result of a blockage to the Eustachian tube. In contrast with adults, the Eustachian tube is shorter and more horizontal in children and also it is consists of more flaccid cartilage, which can impair its opening, therefore otitis media is more common in children ${ }^{(1)}$. Recurrent Acute otitis media (RAOM) causes pain and discomfort in children, also it was noted that 20

\section{Corresponding Author:}

Kais Kassim Ghaima

Assistant Professor, Institute of Genetic Engineering and Biotechnology for Postgraduate Studies, University of Baghdad, Baghdad, Iraq e-mail: kaiskassim@gmail.com
$30 \%$ of infants suffer from RAOM and approximately $70 \%$ of infants experience at least one otitis episode by the age of 2 years ${ }^{(2)}$. Multiple otopathogens can colonize the middle ear and nasopharyngeal. The bacterial attachment and colonization, biofilm formation, and invasion of the middle ear are enhanced by the viral infections of the nasopharynx ${ }^{(3)}$. The main pathogenic bacteria which contribute with the middle ear infections are Haemophilus influenzae, Streptococcus pneumoniae, Streptococcus pyogenes, and Moraxella catarrhalis) and considered as the main risk for OM and RAOM ${ }^{(4,5)}$. Several studies indicated to other species whic isolated from the middle ear fluid of children as causative agents of chronic suppurative otitis media(CSOM) such as Pseudomonas aeruginosa, Staphylococcus aureus, Staphylococcus epidermidis, Klebsiella spp and Escherichia coli ${ }^{(6,7)}$. Most of the bacterial pathogens can form biofilm as the main step for colonization and this biofilm provide some bacteria more effective resistance and tolerance to antibiotics according to the 
complicated structure of bacterial biofilm ${ }^{(8)}$. In Iraq, there is little attention about the study of the role of bacteria species in Otitis media occurrence and their capacity to the formation of biofilm and its relation to increasing antibiotic resistance. Therefore the aims of the study are: Isolation and identification of some species of bacteria, which infect the Iraqi children with otitis media infection by using conventional and molecular method. Investigate the antibiotic resistance of these isolates and study their ability to form the biofilm as a virulence factor. Also, conducting the antibiotics synergism to detection the lowest concentrations of the combination of antibiotics to avoid resistance of bacteria.

\section{Materials and Method}

The samples of the study: The collection of study samples has taken place at the period between November 2018 and completed at end of April 2019, it has included 138 clinical specimens as ear discharge samples, collected from inpatients and outpatients with otitis media infections that admitted in four hospitals in Baghdad, from both gender with age ranging from 6 months to 15 years.

Isolation and identification of bacteria: The bacterial which selected for this study were identified by biochemical tests and Vitek2 system, these isolates include 28 of pathogenic bacterial isolates distributed as 7 from 4 species (Pseudomonas aeruginosa, Proteus mirabilis, Klebsiella pneumoniae and Staphyllococcus aureus) for Quantification of biofilm formation. From the same group of bacteria, 18 multidrug resistant isolates of three bacteria species (Pseudomonas aeruginosa, Proteus mirabilis, and Klebsiella pneumoniae) were selected for evaluation the antibiotics combination

Antibiotics Synergy Test by Using Checkerboard Method: Checkerboard is method to evaluate interactions between antibiotics with serial concentrations as synergism, additive, indifferent, or antagonism ${ }^{(9)}$.

Amikacin, Ciprofloxacin and Ceftazidime, each antibiotic is prepared and MICs are calculated for each antibiotic by microdilution method by using resazurin dye as described by Elshikh et al. (2016) ${ }^{(10)}$.

The checkerboard method uses for study Synergism between Ceftazidime - Ciprofloxacin and Ceftazidime - Amikacin. The test done for most prevalent three bacterial species with the highest antibiotic resistance, which detected in otitis media cases. The concentrations were from $(1 \mu \mathrm{L}-64 \mu \mathrm{L})$ for Ceftrazidime, $(0.5 \mu \mathrm{L}$ $256 \mu \mathrm{L}$ ) for Amikacin and Ciprofloxacin. Dilution chequerboard technique was conducted to evaluate the effect of antibiotic combinations. Fractional inhibitory concentration (FIC) indices for each agent is calculated by dividing the MIC of the antibiotics when used in combination by that of the drug alone. The FIC index is the sum of the FICs of each of two antibiotics when examined in combination ${ }^{(11)}$.

A minimum FIC index of $\leq 0.5$ indicates synergy, while, if the minimum FIC index is $>0.5$ and $\leq 1$, the effect of the combination was classified as additive. If the minimum FIC index is $>1$ and $\leq 2$, the effect of the combination was classified as indifferent, and antagonistic if $>2^{(9)}$.

Microtitre plate assay for biofilm quantification: Biofilm was formed on 96 well flat bottom polystyrene microtitre plates as described by Kurmusaoglu (2019) ${ }^{(12)}$. Briefly, A $10 \mu \mathrm{l}$ of cell suspension having 0.5 O.D600 nm was inoculated in $190 \mu$ Tryptic soy broth medium in each well. Tow hundred $\mu 1$ of sterile distilled water was added in peripheral wells to reduce the water loss. Then microtitre plate was incubated at $37^{\circ} \mathrm{C}$ for $18 \mathrm{~h}$. After aspiration of planktonic cells, biofilms were fixed with $99 \%$ methanol. Plates are washed twice with phosphate buffer saline or sterile saline water and air dried. Then, $200 \mu \mathrm{l}$ of crystal violet solution $(0.2 \%)$ was added to all wells and the excess crystal violet was removed after 5 min, and plates were washed twice and air dried. The cell bound crystal violet was dissolved in $33 \%$ acetic acid. Biofilm formation was measured in terms of O.D $570 \mathrm{~nm}$ using micro plate reader. Cut off value (ODc) was calculated, which can provide categorization of isolates as biofilm producer or not.

Statistical Analysis: The Statistical Analysis System, SAS (2012) ${ }^{(13)}$ program used in this study. According to Chi-square test uses for significant comparing between percentages. Least significant difference (LSD) test was used to significant compare between means ( 0.05 and 0.01 probability) in this study

\section{Results and Discussion}

The checkerboard method uses for studying Synergism between Ceftazidime- Ciprofloxacin and Ceftazidime - Amikacin. The test is done for most prevalent three bacterial species with the highest antibiotic resistance, which detected in otitis media infections. The concentrations are from $(1-64 \mu \mathrm{g} / \mathrm{ml})$ for Ceftrazidime, 
$(0.5-256 \mu \mathrm{g} / \mathrm{ml})$ for Amikacin and Ciprofloxacin. The results of MICs of three antimicrobial agents and FICs of 2 combinations against 18 isolates of three bacteria species (Pseudomonas aeruginosa, Proteus mirabilis, and Klebsiella pneumoniae) are showed in Table (1).

Table (1). MIC of antibiotic combinations and synergy test results.

\begin{tabular}{|c|c|c|c|c|c|c|c|}
\hline Isolates & $\begin{array}{l}\text { Antibiotics } \\
\text { combination }\end{array}$ & $\begin{array}{c}\text { MIC of first } \\
\text { antibiotic alone } \\
\qquad(\mu \mathrm{g} / \mathrm{ml})\end{array}$ & $\begin{array}{c}\text { MIC of first } \\
\text { antibiotic in } \\
\text { combination }(\mu \mathrm{g} / \mathrm{ml})\end{array}$ & $\begin{array}{l}\text { MIC of second } \\
\text { antibiotic alone } \\
\qquad(\mu \mathrm{g} / \mathrm{ml})\end{array}$ & $\begin{array}{l}\text { MIC of second } \\
\text { antibiotic in } \\
\text { combination } \\
(\mu \mathrm{g} / \mathrm{ml})\end{array}$ & FIC & Result \\
\hline A1 & AK-CAZ & 64 & 16 & 64 & 8 & 0.375 & Synergy \\
\hline A1 & CIP-CAZ & 4 & 4 & 64 & 16 & 1.25 & Indifferent \\
\hline A5 & AK-CAZ & 8 & 2 & 128 & 32 & 0.5 & Synergy \\
\hline A5 & CIP-CAZ & 32 & 8 & 128 & 32 & 0.5 & Synergy \\
\hline A27 & AK-CAZ & 64 & 16 & 64 & 8 & 0.375 & Synergy \\
\hline A27 & CIP-CAZ & 4 & 2 & 64 & 16 & 0.75 & Additive \\
\hline $\mathrm{P} 1$ & AK-CAZ & 32 & 4 & 32 & 8 & 0.375 & Synergy \\
\hline P1 & CIP-CAZ & 2 & 1 & 32 & 16 & 1.0 & Additive \\
\hline $\mathrm{P} 20$ & AK-CAZ & 64 & 16 & 64 & 16 & 0.5 & Synergy \\
\hline $\mathrm{P} 20$ & CIP-CAZ & 32 & 32 & 64 & 32 & 1.5 & Indifferent \\
\hline P23 & AK-CAZ & 32 & 4 & 32 & 16 & 0.625 & Additive \\
\hline P23 & CIP-CAZ & 64 & 16 & 32 & 8 & 0.5 & Synergy \\
\hline K4 & AK-CAZ & 64 & 16 & 16 & 4 & 0.5 & Synergy \\
\hline $\mathrm{K} 4$ & CIP-CAZ & 16 & 4 & 16 & 8 & 0.75 & Additive \\
\hline K14 & AK-CAZ & 128 & 64 & 64 & 32 & 1 & Additive \\
\hline K14 & CIP-CAZ & 32 & 8 & 64 & 8 & 0.375 & Synergy \\
\hline K15 & AK-CAZ & 128 & 32 & 64 & 16 & 0.5 & Synergy \\
\hline K15 & CIP-CAZ & 16 & 4 & 64 & 16 & 0.5 & Synergy \\
\hline
\end{tabular}

FIC: Fractional Inhibitory Concentration; AK: Amikacin; CAZ: Ceftazidime; CIP: Ciprofloxacin; A= Pseudomonas aeruginosa, P = Proteus mirabilis, $\mathrm{K}=$ Klebsiella pneumoniae.

Results revealed combinations ceftazidimeamikacin and ceftazidime- ciprofloxacin displayed synergistic activity (FIC $\leq 0.5)$ against the most of tested isolates except tow isolates $P$. aeruginosa A 1 and $P$. mirabilis $\mathrm{P} 20$, which showed indifferent with the ceftazidime-ciprofloxacin combination.
In the current study, none of the antimicrobial combinations tested demonstrated antagonism against any of the isolates tested. Out of 18 isolates, 11 exhibited synergistic activities, while the additive activity showed in 5 isolates, as the result of $K$. pneumoniae K4 (Figure 1). 


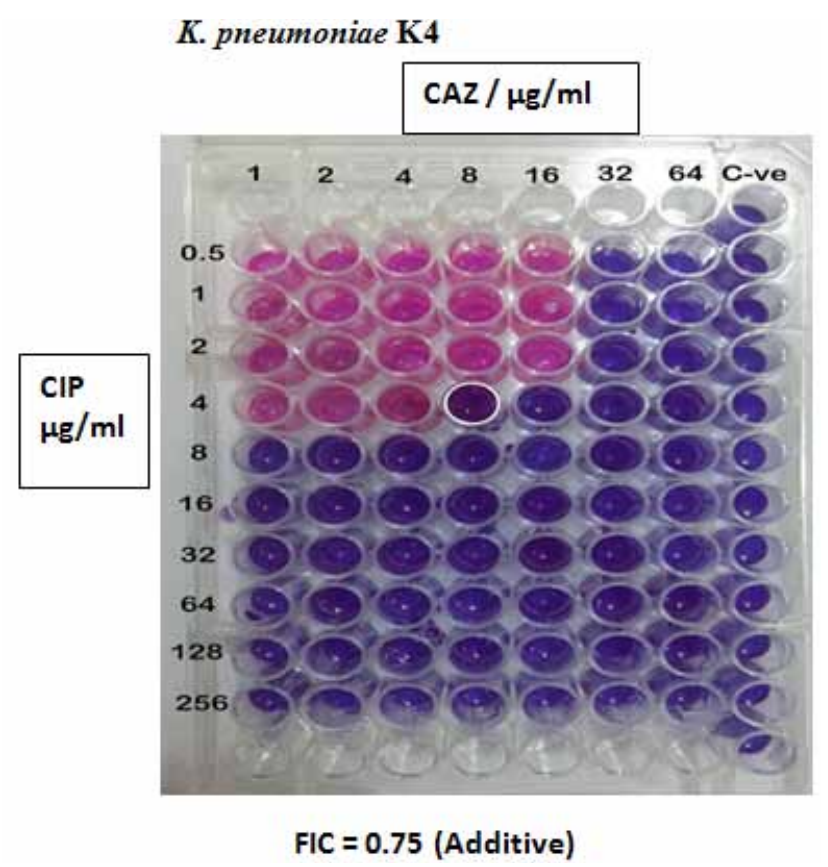

Figure (1). FIC of antibiotics combination ceftazidime-amikacin against $K$. pneumoniae $\mathrm{K4}$.

The four isolates $P$. aeruginosa A1, A27, $P$. mirabilis $\mathrm{P} 1$, and $K$. pneumoniae $\mathrm{K} 14$ displayed the highest affected by the 2 combinations especially with ceftazidime-amikacin $($ FIC $=0.375)($ Table 1$)$.

Antibiotic combinations to the treatment the resistant infections, have been reported to be effective. One drug may overwhelm or neutralize the mechanisms of bacterial resistance, repurposing the antibiotic drug by increasing its efficacy. May be the best-known example of antibiotic synergy is the combination of clavulanic acid with $\beta$-lactam antibiotics ${ }^{(14)}$. Antibiotic combination therapy is used to prevent the emergence of resistant microbial strains, treat emergency cases during the process of etiological diagnosis, and to take advantage of antibiotic synergism. This is noted to be effective when two bactericidal agents are combined, but antagonism occurs in vitro on combination of a bacteriostatic and a bactericidal antibiotic ${ }^{(15)}$ checkerboard technique is an in vitro synergy test using to determine the antimicrobial combinations activity. It is a simple to perform and remains to be a widely used technique to assess antimicrobial combinations ${ }^{(16)}$. Aminoglycoside and beta-lactam combinations are the most frequent used for the treatment of many infections especially due to $P$. aeruginosa. Many studies reported these synergistic combinations ${ }^{(17,18)}$. In this study, it is detected that the synergy was obvious between Ceftazidime and Amikacin against $P$. aeruginosa isolates. Also, many reports indicated to the combinations of quinolone and beta-lactam antibiotics and the synergism with several rates was observed $^{(19,20)}$. In this study, synergy was less effective in resistant strains with Ceftazidime-ciprofloxacin combination. Mayer and Nagy (1999) ${ }^{(19)}$ revealed that the combination of third-generation cephalosporin with fluoroquinolones rarely shows synergy. In agreement with the results of current study, Hannan et al. (2014) (21) showed that among the four combinations evaluated ceftazidime-ceftazidime-meropenem, piperacillin/ tazobactam, ceftazidime-amikacin and ceftazidimeciprofloxacin, ceftazidime-amikacin was in high rates of synergy in multidrug resistant strains of $P$. aeruginosa. Combinations of aminoglycosides and $\beta$-lactams have synergic effects against gram negative isolates of this study. The mechanism of this combination may be due to the action of $\beta$-lactams on bacterial cell wall by destruction of peptidoglycan polymers and then easily of the entry of the aminoglycosides into the bacterial cell (22).

Out of the total isolates of OM infections, 28 isolates with high antibiotic resistance are selected for detection the Quantitative biofilm formation by Tissue Culture Plate (TCP) method, these isolates were distributed as 7 from 4 species which include (Pseudomonas aeruginosa, Proteus mirabilis, Klebsiella pneumoniae and Staphyllococcus aureus). Quantification of biofilm formation has been shown in Table (2) and Figure (2).

All isolates $(100 \%)$ showed biofilm production by TCP method. Strong biofilm producers were 14 (50\%), $10(35.7 \%)$ were moderate and $4(14.2 \%)$ isolates were considered as weak biofilm producers. In this study, the strong biofilm production is the most prevalent in $P$. aeruginosa $(7,100 \%)$ followed by $S$. aureus $(5,71.4 \%)$, while the most of $P$. mirabilis and $K$. pneumoniae are moderate biofilm producers. 
Table (2). Screening of OM pathogenic isolates for biofilm formation by Tissue Culture Plate method.

\begin{tabular}{|c|c|c|c|c|c|c|}
\hline \multirow{2}{*}{$\begin{array}{l}\text { BACTERIAL } \\
\text { SPECIES }\end{array}$} & \multirow{2}{*}{$\begin{array}{l}\text { Number of } \\
\text { investigate } \\
d \text { isolates } \\
(\%)\end{array}$} & \multicolumn{4}{|c|}{ BIOFILM PRODUCTION } & \multirow{2}{*}{$\begin{array}{l}\text { Chi- } \\
\text { Square } \\
\left(x^{2}\right)\end{array}$} \\
\hline & & STRONG & MODERATE & WEAK & $\begin{array}{l}\text { NON } \\
\text { PRODUCTION }\end{array}$ & \\
\hline $\begin{array}{l}\text { Pseudomonas } \\
\text { aeruginosa }\end{array}$ & 7 & 7 & 0 & 0 & 0 & $6.34^{* *}$ \\
\hline $\begin{array}{l}\text { Proteus } \\
\text { mirabilis }\end{array}$ & 7 & 1 & 4 & 2 & 0 & $2.08 \mathrm{NS}$ \\
\hline $\begin{array}{l}\text { Klebsiella } \\
\text { pneumoniae }\end{array}$ & 7 & 1 & 5 & 1 & 0 & $4.52 *$ \\
\hline $\begin{array}{l}\text { Staphylococcus } \\
\text { aureus }\end{array}$ & 7 & 5 & 1 & 1 & 0 & $4.71 *$ \\
\hline Total & $28(100 \%)$ & $\begin{array}{c}14 \\
(50 \%)\end{array}$ & $\begin{array}{c}10 \\
(35.7 \%)\end{array}$ & $\begin{array}{c}4 \\
(35.7 \%)\end{array}$ & 0 & $6.41 * *$ \\
\hline
\end{tabular}

$*(\mathrm{P}<0.05),{ }^{* *}(\mathrm{P}<0.01)$.

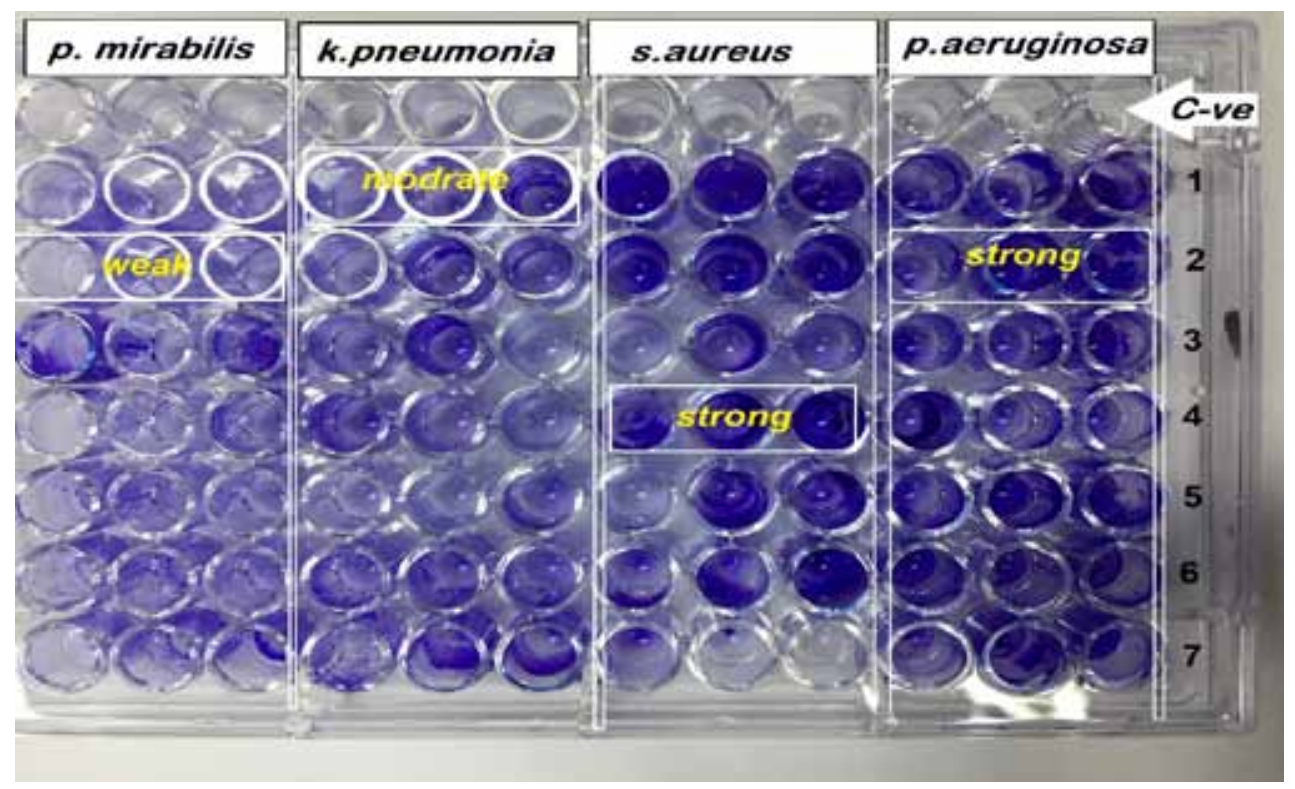

Figure (2). Detection of biofilm producers of otitis media bacterial pathogens by Tissue Culture Plate method with crystal violet staining.

In this study, the unique ability of $P$. aeuginosa to form biofim makes it as the main cause of OM infections in Iraqi children, where, Chronic suppurative otitis media is largely due to biofilm-forming bacteria, of which a common pathogen is Pseudomonas aeruginosa, and this species develop into biofilms and form chronic infections in the middle ear of a mouse model of Eustachian tube obstruction and acute tympanic membrane wounds ${ }^{(23)}$.

The weak biofilm formation of $P$. mirabilis and $K$. pneumoniae in $\mathrm{OM}$ infection may be due to that these bacteria related to bacterial colonization of catheter devices infections such as urinary tract infections ${ }^{(24)}$. It finds that the mucosal biofilm has been implicated in several pediatric respiratory infections, including otitis media with effusion, tonsillitis, adenoiditis, persistent endobronchial infections, chronic rhinosinusitis, and bronchiectasis ${ }^{(25)}$. The nasopharyngeal biofilms have the main role in the effectiveness of antibiotic in children with otitis media, because the biofilm play a role in the development of chronic nasopharyngeal inflammation, which is may be associated with chronic 
or recurrent middle ear disease ${ }^{(26)}$. The bacterial biofilms of clinically $H$. influenzae in patients with acute otitis media infections are responsible for the development of acute middle ear infections on the basis of the detection of biofilms produced by this species ${ }^{(27)}$. The production of biofilm may explain the failure of traditional antibiotic treatment acute otitis media, which act as favourable environment ${ }^{(28)}$. Biofilm formation is considered as a survival strategy by bacteria to antibiotics which are effective against bacteria. Biofilms are almost impossible to grow in the laboratory media and are incredibly resistant to antimicrobials, which mean that the diagnosis of chronic OM is one of the most challenging in the management of middle-ear infection ${ }^{(29)}$. The previous results gave a role of biofilm formation in the entire cavity of the middle ear of children with recurrent AOM, contributing to the viscosity of effusion ${ }^{(30)}$.

\section{Conclusion}

The current study revealed that the strong biofilm was the most prevalent in P. aeruginosa followed by $S$. aureus and the biofilm formation leads to difficulty in treatment of OM, mainly due to its frequent association with multidrug resistant bacteria, also, it was obvious that the Combinations ceftazidime-amikacin and ceftazidime-ciprofloxacin displayed synergistic activity against the most of tested isolates.

Source of Funding: Authors

Ethical Clearance: Yes

\section{Conflict of Interest: Nil}

\section{References}

1. Biradar SK. Study of Microbiological Profile and their Antibiogram in Patients with Chronic Suppurative Otitis Media. IJCMAS. 2015; 4(9): 981-85.

2. Klein JO. The burden of otitis media. Vaccine. 2000; 19: S2-S8.

3. Murphy TF, Chonmaitree T, Barenkam S, Kyd J, Nokso-Koivisto J, Patel JA. Microbiology and immunology panel. Otolaryngol Head Neck Surg. 2013; 148(4): 64-89.

4. Pelton SI, Leibovitz E. Recent advances in otitis media. The Pediatric Infectious Disease Journal. 2009; 28(10): S133-S137.

5. Pichichero ME. Ten-year study of acute otitis media in Rochester, NY. The Pediatric Infectious Disease Journal. 2016; 35(9): 1027-1032.

6. Ngo C, Maasa H, Thorntion R, Cripps A. Predominant bacteria detection from the middle ear fluid of Children experiencing Otitis media: A systematic review . Pols One . 2016; 11: e0150949.

7. Toman J, Moll A, Barnes M, Shenoi S, Porterfield JZ. The Role of Routine Culture in the Treatment of Chronic Suppurative Otitis Media: Implications for the Standard of Care in Rural Areas of South Africa. Tropical medicine and infectious disease. 2019; 4(1): 10.

8. Kumar A, Alam A, Rani M, Ehtesham NZ, Hasnain SE. Biofilms: Survival and defense strategy for pathogens. International Journal of Medical Microbiology. 2017; 307(8): 481-489.

9. Rizvi M, Ahmad J, Khan F, Shukla I, Malik A, Sami H. Synergy of drug combinations in treating multidrug-resistant Pseudomonas aeruginosa. The Australasian Medical Journal. 2015; 8(1): 1.

10. Elshikh M, Ahmed S, Funston S, Dunlop P, McGaw M, Marchant R. et al. Resazurin-based 96-well plate microdilution method for the determination of minimum inhibitory concentration of biosurfactants. Biotechnol Lett. 2016; 38:10151019.

11. Nakamura A, Hosoda M, Kato T, Yamada Y, Itoh M, Kanazawa K, Nouda H. Combined effects of meropenem and aminoglycosides on Pseudomonas aeruginosa in vitro. Journal of Antimicrobial Chemotherapy. 2000; 46(6): 901-904.

12. Kirmusaoglu S. The Method for Detection of Biofilm and Screening Antibiofilm Activity of Agents. In: Exopolysaccharides-Method of Preparation and Application. IntechOpen, 2019.

13. SAS. Statistical Analysis System, Useres Guide. Statistical. Version 9.1 th ed. SAS. Inst. Inc. Cary. N.C. USA, 2012.

14. Holt KE, Wertheim H, Zadoks RN, Baker S, Whitehouse CA, Dance D. et al. Genomic analysis of diversity, population structure, virulence, and antimicrobial resistance in Klebsiella pneumoniae, an urgent threat to public health. Proceedings of the National Academy of Sciences. 2015; 112(27): E3574-E3581.

15. Daschner FD. Combination of bacteriostatic and bactericidal drugs: lack of significant in vitro antagonism between penicillin, cephalothin, 
and rolitetracycline. Antimicrobial Agents and Chemotherapy. 1976; 10(5): 802-808.

16. Vardakas K Z, Athanassaki F, Pitiriga V, Falagas ME. Clinical relevance of in vitro synergistic activity of antibiotics for multidrug-resistant Gramnegative infections: A systematic review. Journal of Global Antimicrobial Resistance. 2019; 17: 250259.

17. Sader HS, Huynh HK, Jones RN. Contemporary in vitro synergy rates for aztreonam combined with newer fluoroquinolones and $\beta$-lactams tested against gram-negative bacilli. Diagnostic Microbiology and Infectious Disease. 2003; 47(3): 547-550.

18. Song W, Woo HJ, Kim JS, Lee KM. In vitro activity of $\beta$-lactams in combination with other antimicrobial agents against resistant strains of Pseudomonas aeruginosa. International Journal of Antimicrobial Agents. 2003; 21(1): 8-12.

19. Mayer I, Nagy E. Investigation of the synergic effects of aminoglycoside-fluoroquinolone and third-generation cephalosporin combinations against clinical isolates of Pseudomonas spp. Journal of Antimicrobial Chemotherapy. 1999; 43(5): 651-657.

20. Fish DN, Choi MK, Jung R. Synergic activity of cephalosporins plus fluoroquinolones against Pseudomonas aeruginosa with resistance to one or both drugs. Journal of Antimicrobial Chemotherapy. 2002; 50(6): 1045-1049.

21. Hannan A, Gondal AJ, Saleem S, Arshad MU, Lateef W. In-vitro efficacy of ceftazidime in combinations with meropenem, piperacillin/ tazobactam, amikacin and ciprofloxacin against extensively drug resistant Pseudomonas aeruginosa. Pakistan J Zool. 2014; 46: 1111-1116.

22. Watanabe T, Obayashi K, Matsui K, Kubota T. Comparative studies of the bactericidal morphological and post-antibiotic affects of arbekacin and vancomycin against methicillinresistant Staphylococcus aureus. Journal of Antimicrobial Chemotherapy. 1997; 39: 471-6.

23. Khomtchouk KM, Kouhi A, Santa Maria P. The Role of Neutrophils in Pseudomonas aeruginosa Chronic Suppurative Otitis Media. J Immunol. 2019; 202 (1 Supplement): 190.

24. Verma A, Bhani D, Tomar V, Bachhiwal R, Yadav S. Differences in bacterial colonization and biofilm formation property of uropathogens between the two most commonly used indwelling urinary catheters. Journal of Clinical and Diagnostic Research: JCDR. 2016; 10(6): PC01 -PC3.

25. Hamilos DL. Biofilm Formations in Pediatric Respiratory Tract Infection. Current Infectious Disease Reports. 2019; 21(2): 6.

26. Torretta S, Drago L, Marchisio P, Ibba T, Pignataro L. Role of Biofilms in Children with Chronic Adenoiditis and Middle Ear Disease. Journal of Clinical Medicine. 2019; 8(5): 671.

27. Moriyama S, Hotomi M, Shimada J, Billal DS, Fujihara K, Yamanaka N. Formation of biofilm by Haemophilus influenzae isolated from pediatric intractable otitis media. Auris Nasus Larynx. 2009; 36(5): 525-531.

28. Rayner MG, Zhang Y, Gorry MC, Chen Y, Post JC, Ehrlich GD. Evidence of bacterial metabolic activity in culture-negative otitis media with effusion. JAMA. 1998; 279(4): 296-299.

29. Abu Bakar MB, McKimm J, Haque M. Otitis media and biofilm: An overview. Int J Nutr Pharmacol Neural Dis. 2018; 8: 70-78.

30. Thornton RB, Wiertsema SP, Kirkham LAS, Rigby PJ, Vijayasekaran S, Coates HL. Neutrophil Extracellular Traps and Bacterial Biofilms in Middle Ear Effusion of Children with Recurrent Acute Otitis Media, A Potential Treatment Target. PLoS ONE. 2013; 8(2): e53837. 